\title{
Prevalencia de violencia de pareja en mujeres estudiantes de una universidad pública
}

Pedroza-Roque María Guadalupe*, Mora-Santoyo María Isabel*, García-Aquino Guadalupe del Carmen*, Ruvalcaba-González Alejandra Guadalupe*, González-Flores Silvia Patricia**, Rodríguez-Medina María de Lourdes**

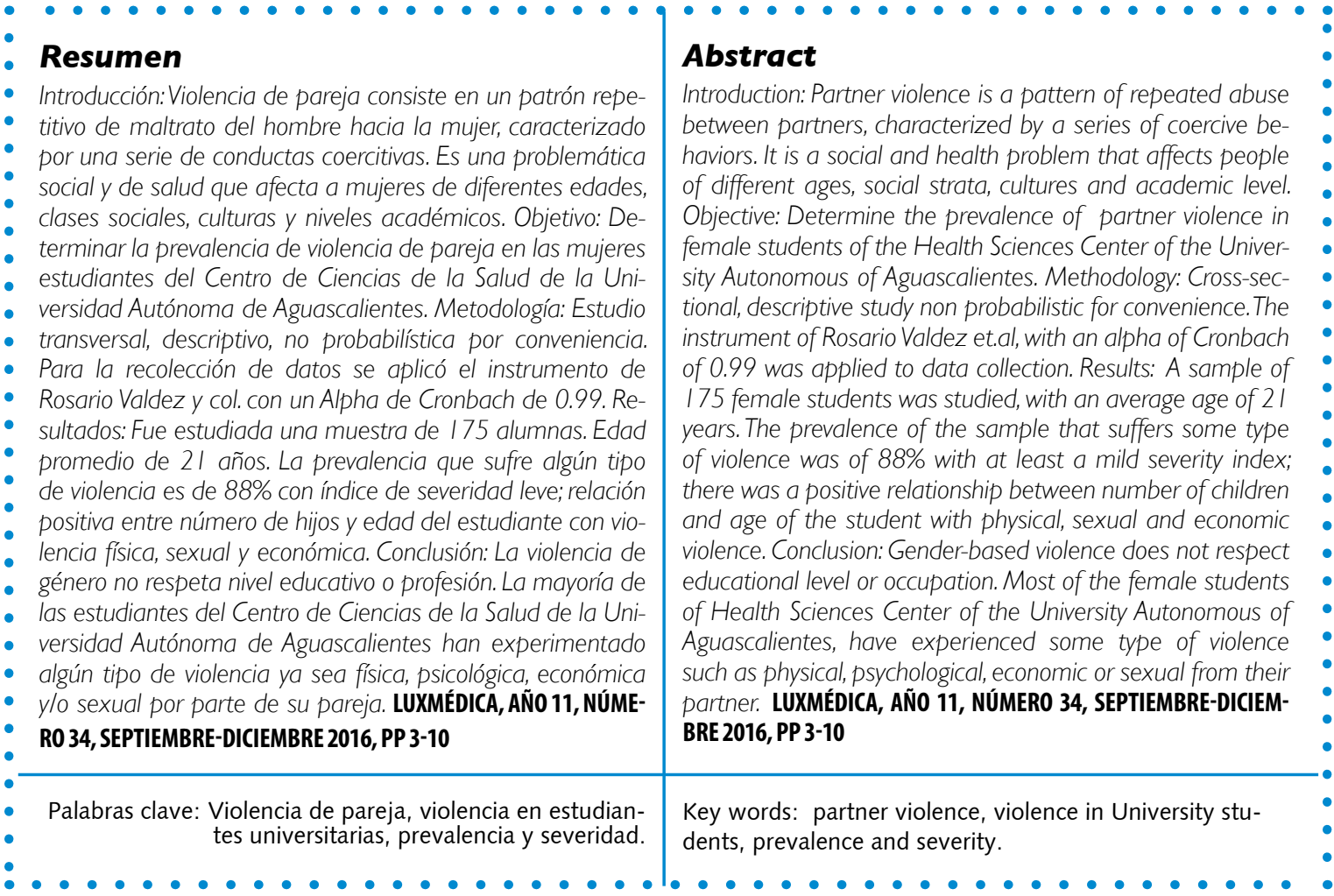

* Universidad Autónoma de Aguascalientes. Departamento de enfermería, estudiantes de la Licenciatura de Enfermería del Centro de Ciencias de la Salud.

** Universidad Autónoma de Aguascalientes Maestras en Ciencias de la Enfermería. Profesoras investigadoras del Departamento de Enfermería del Centro de Ciencias de la Salud.

Fecha de recibido: 15 mayo 2016

Fecha de aceptación: 13 de agosto 2016

Correspondencia: MCE María de Lourdes Rodríguez Medina. Departamento de Enfermería del Centro de Ciencias de la Salud Universidad Autónoma de Aguascalientes. Edificio 105. Avenida Universidad № 940 Ciudad Universitaria. Código postal 20131. Aguascalientes, Ags., México. Teléfono 9108437, correo electrónico: Iulardz_del@hotmail.com 


\section{Introducción}

La violencia de género es una problemática social y de salud que afecta a mujeres de diferentes edades, clases sociales, culturas y niveles académicos. Se sabe que desde tiempos remotos ha existido violencia y discriminación hacia la mujer. ${ }^{1}$ En países orientales, se considera a la mujer como un objeto de posesión del hombre, que puede hacer con ellas lo que desee una vez que se casan. Aún en la actualidad muchas de estas creencias se mantienen y no sólo en tribus o países tercer mundistas, sino también en EE.UU. o China, países considerados de primer nivel. ${ }^{2}$ La violencia de género representa el instrumento de sometimiento por excelencia en el sistema patriarcal, ejerciendo la dominación y control, perpetuando la supremacía masculina y la subordinación femenina. ${ }^{3}$ A partir de los últimos veinte años se produce un cambio y se empieza a reconocer como un problema social, como un problema público y político, que hay que erradicar, ya que ataca directamente a los derechos humanos, que atenta contra la estabilidad, el desarrollo económico y social de los pueblos, y al principio de igualdad de oportunidades que debe establecerse en toda sociedad democrática. ${ }^{4}$

La violencia consiste en un patrón repetitivo de maltrato por parte de la pareja masculina hacia la mujer, caracterizado por una serie de conductas coercitivas que pueden incluir: violencia física, violencia emocional, violencia sexual, violencia económica. ${ }^{5}$ La violencia se considera un problema de salud pública, no sólo porque tiene consecuencias en la salud de la mujer, sino también porque los servicios sanitarios son los lugares donde más frecuentemente acuden las mujeres en esta situación. ${ }^{6}$

A partir del reconocimiento de que la violencia es un asunto de todos, que además "duele, lacera, lastima a unos y preocupa a otros", surge la necesidad de realizar la observación de ésta desde una actitud crítica, holística e integral de búsqueda permanente, en la que la violencia de género sea dimensionada, nunca ocultada.

De 24 millones de mujeres colombianas de 15 y más años, casadas o unidas, el $44.9 \%$ aceptaron haber sido víctimas de violencia por parte de su pareja a lo largo de su relación. ${ }^{6}$

Ninguna característica socio demográfica puede definir a las mujeres violentadas, ya que el maltrato a las mujeres por parte de su pareja ocurre en cualquier ámbito social, económico y cultural. En este sentido esta investigación contribuirá a proporcionar datos y determinar la violencia de género que sufren las estudiantes de la licenciatura de enfermería del Centro de Ciencias de la Salud y, una vez identificada, se mostrarán los resultados para sugerir programas y estrategias de promoción y educación para la salud ya que son unos de los principales objetivos de la enfermería: educar y promover la salud. 


\section{Material y métodos}

Estudio con enfoque cuantitativo, descriptivo, observacional, prospectivo, transversal, analítico. Realizado en un tiempo aproximado de 2 años comprendiendo desde la elaboración del protocolo hasta la difusión de los resultados. Participaron mujeres estudiantes de la Universidad Autónoma de Aguascalientes del Centro de Ciencias de la Salud, con una edad aproximada de entre 17 a 51 años. Las cuales fueron seleccionadas mediante un muestreo no probabilístico por conveniencia, siendo la muestra de 175 estudiantes que cumplieron los criterios de investigación: estar inscrita en alguna licenciatura del Centro de Ciencias de la Salud, que tuvieran una relación de pareja mínimo de 12 meses.

Para la recolección de datos se aplicó el instrumento "Escala de violencia e índice de severidad de violencia de pareja en mujeres mexicanas" el cual cuenta con 21 reactivos con el objetivo de medir distintos grados de violencia en sus diferentes modalidades: violencia física, emocional, sexual y económica con un alpha de Cronbach 0.99. La EV (escala de violencia) se compone de cuatro sub-escalas que miden la frecuencia de las acciones violentas en los últimos 12 meses $(1=$ nunca, $2=$ alguna vez, $3=$ varias veces y $4=$ muchas veces).

Se elaboró una base de datos en el paquete estadístico SPSS 21 en la cual se calcularon las medidas de tendencia central, dispersión, posición y relación de las variables estudiadas. Posteriormente se realizó un análisis de los resultados obtenidos así como el cruce de variables para identificar la relación variables sociodemográficas y tipo de violencia con la prueba estadística Spearman; además, se calculó la severidad.

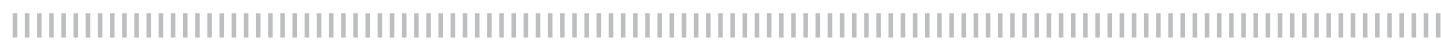

\section{Resultados}

Fue estudiada una muestra de 175 alumnas del Centro de Ciencias de la Salud que cumplieron los criterios de inclusión. La edad promedio de las alumnas fue de 21 años, mínima de 17 y máxima de 51. La edad media de la pareja de las estudiantes fue de 23 años con un mínimo de 18 y un máximo de 60 años.

De las carreras estudiadas, la que cuenta con más mujeres con pareja, según los criterios de Rosales Valdez, fue la licenciatura de enfermería con un porcentaje de $46,3 \%$ (figura 1 ).
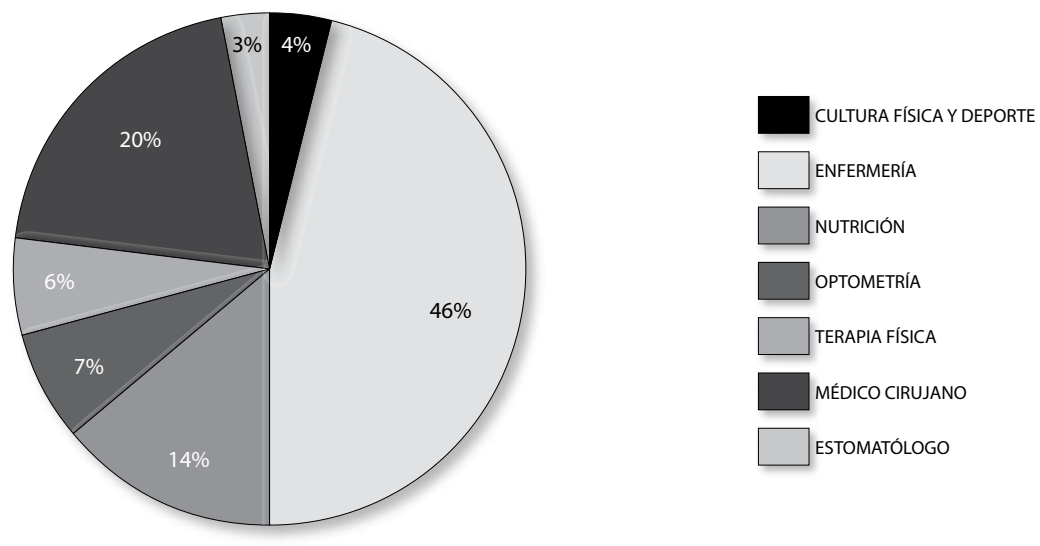

Fuente: Prevalencia de violencia de pareja en mujeres estudiantes de un centro de una universidad pública Figura 1. Distribución de estudiantes encuestadas según la licenciatura en que están inscritas 
Los semestres predominantes donde las estudiantes tienen una relación de pareja son: quinto semestre con el $17.1 \%$, séptimo semestre un $16 \%$ y un $13.7 \%$ el octavo. Con respecto a la ocupación de la muestra estudiada, además de estudiar, trabajan y/o se dedican al hogar un $9 \%$ de las estudiantes. Las parejas de las encuestadas se dedican el $43,4 \%$ al estudio, $36,6 \%$ son empleados y $5.7 \%$, ambos.

En el estado civil de las alumnas $66 \%$ es soltera, $19 \%$ viven en unión libre y $14 \%$ son casadas. El tiempo de relación fue de un mínimo de 12 meses y un máximo de 402 meses (34 años); el 83.4 \% de las es- tudiantes refirieron que no tienen hijos, $11.4 \%$ tiene 1 hijo y sólo $1 \%$ tiene 4 hijos.

En la religión, se encontró que el $85.7 \%$ de las estudiantes manifestaron ser católica, y $6.9 \%$ cristiana. En cuanto el ingreso mensual el promedio es de $\$ 1000$ con un mínimo de $\$ 100$ pesos y un máximo de $\$ 20,000$.

Por consiguiente, de la muestra estudiada sólo 21 estudiantes no sufren ningún tipo de violencia, lo cual equivale al $12 \%$ y el porcentaje restante sufre algún tipo de violencia, del cual 154 alumnas sufren violencia psicológica, 38 violencia física, 11 sexual y 14 violencia económica (figura 2 ).

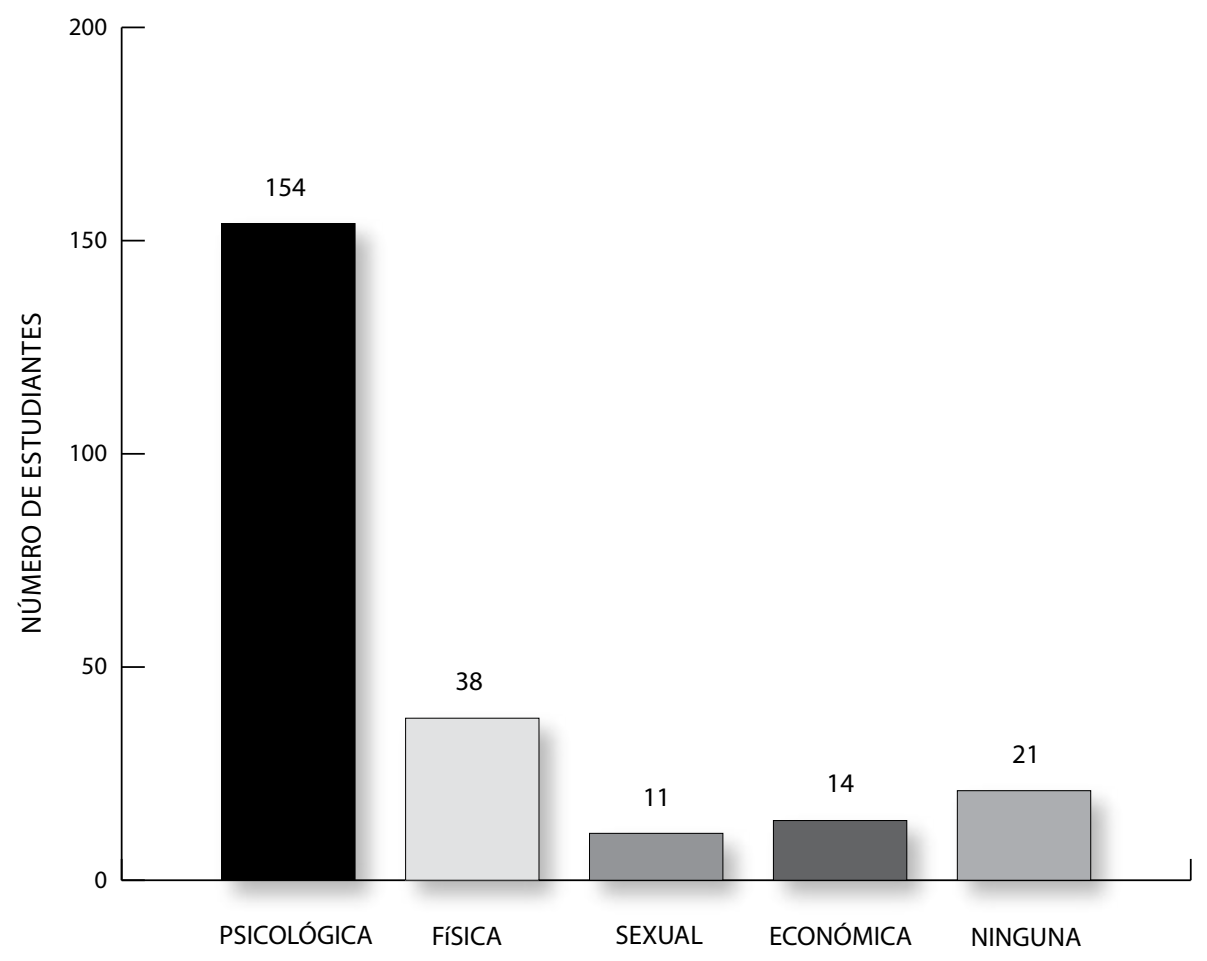

Fuente: Prevalencia de Violencia de Pareja en Mujeres Estudiantes de un Centro de una Universidad Pública

Figura 2. Distribución de la frecuencia, según el tipo de de violencia.

Al relacionar las variables socio-demográficas y el tipo de violencia se observó que existe relación entre la violencia física, sexual y económica con el número de hijos y la edad de la estudiante. Así mismo, el índice de severidad en la violencia psicológica se encuentra mayormente en un nivel leve con un $83 \%$ de los casos, moderado con el $4 \%$ y sólo el $1 \%$ grave, como se muestra en la tabla 1. 


\section{Tabla I}

Frecuencia de la violencia psicológica de acuerdo a su grado de severidad

\begin{tabular}{|c|c|c|c|c|}
\hline \multicolumn{5}{|c|}{ Severidad de la violencia psicológica } \\
\hline & & Valor & Frecuencia & Porcentaje \\
\hline \multirow{4}{*}{$\begin{array}{l}\text { VIOLENCIA } \\
\text { PSICOLÓGICA }\end{array}$} & NO PROBLEMA & 0 & 21 & 12 \\
\hline & LEVE & 1 a 5 & 146 & 83.42 \\
\hline & MODERADO & 6 a 10 & 7 & 4 \\
\hline & GRAVE & 11 a 15 & 1 & 1 \\
\hline
\end{tabular}

En cuanto a la violencia física, el $22 \%$ tiende a ser un problema leve. En la violencia sexual el $5 \%$ muestra un problema leve y el $2 \%$ un nivel de severidad de violencia sexual grave. Respecto a la violencia económica, encontramos el $92 \%$ de los casos con no problema, $6 \%$ con problema leve, $1 \%$ con moderado y $1 \%$ de severidad. Por lo tanto, de acuerdo al índice de severidad de la violencia, el $88 \%$ de las estudiantes encuestadas han sufrido violencia a nivel leve en algún momento de su relación y sólo el $12 \%$ de éstas se encuentra en no problema, lo que es preocupante y refleja el alto índice de violencia entre las estudiantes del Centro de Ciencias de la Salud de la Universidad Autónoma de Aguascalientes.

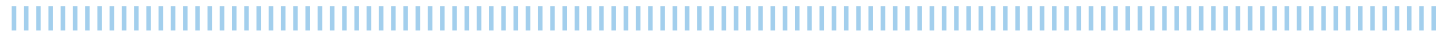

\section{Discusión}

En la Encuesta Nacional sobre Violencia contra las Mujeres 2006 (ENVIM 2006) se informa que tres de cada 10 mujeres sufren violencia de la pareja actual, cuatro de cada 10 mujeres han sufrido violencia de pareja alguna vez en la vida y seis de cada 10 mujeres han vivido violencia alguna vez en la vida, nuestros resultados concuerdan ya que al menos 8 de cada 10 estudiantes han sufrido violencia en su relación de noviazgo.

Rodríguez Borrego ${ }^{3}$ considera este tipo de violencia como un problema de salud pública, no sólo porque tiene consecuencias sobre la salud de la mujer, sino también porque los servicios sanitarios son los lugares donde más frecuentemente acuden las mujeres en esta situación. Entonces, si los resultados demuestran que también existe violencia en el personal que laborará en los centros comunitarios, la cuestión es cómo se espera que las mujeres se sientan seguras y orientadas al acudir a ellos, si son estos mismos los que también la están sufriendo. Calvo González y cols ${ }^{8}$ mencionan que el perfil de las mujeres afectadas se relaciona con un nivel socioeconómico y educativo bajo; los resultados que obtuvimos demuestra que la violencia también las reciben las mujeres con un nivel de educación superior.

En otro estudio de Rodríguez Borrego ${ }^{2}$ publica que la violencia por el compañero íntimo ( $\mathrm{VCl}$ ) comienza con abuso emocional. Ese comportamiento puede derivar para agresión física o sexual. Varios tipos de $\mathrm{VCl}$ pueden suceder concomitantemente. Como se observó del $88 \%$ del total de estudiantes que sufrían violencia, todas éstas recibían, por lo menos, violencia psicológica, lo que podría dar por entendido que es donde inicia, ya que las que llegaron a sufrir algún caso de violencia física, sexual o económica nunca fue de manera aislada, siempre iba acompañada de violencia psicológica. La Organización Mun- 
dial de la Salud (OMS) en su reporte Multi-Country Study of Women's Health and Domestic Violence Against Women, indica variaciones significativas en la prevalencia de la $\mathrm{VCl}$ a lo largo de la vida, entre países y regiones, variando de $13 \%$ a $71 \%$. Esa población necesita de atención de la salud. Los resultados de la prevalencia de violencia de pareja en esta investigación fueron más altos y es de extrema importancia la atención y orientación de la salud.

En un estudio realizado por Vázquez y $\operatorname{cols}^{10}$ reportan que la violencia contra la pareja es un problema frecuente en las jóvenes universitarias, pues encontraron que casi la mitad de las jóvenes fueron maltratadas por sus parejas. De acuerdo a nuestros resultados, la mayoría de las estudiantes que fueron encuestadas han recibido algún tipo de violencia por parte de su pareja en algún momento de sus vidas. También, con la revisión que hacen en ese artículo, afirman que en diversos países la violencia entre parejas de adolescentes y jóvenes es tan frecuente o más que en las parejas adultas; de hecho, las jóvenes de 24 años o menores son las que tienen un mayor riesgo de sufrir violencia por parte de la pareja. Los resultados de este trabajo son acordes con ellos, pues la media de edad de las mujeres incluidas en nuestra investigación fue de 21.6 años.

García Díaz y cols ${ }^{11}$ afirman que la prevalencia de situaciones de maltrato entre las futuras enfermeras es elevada; en este estudio, el $46.3 \%$ que contestaron los instrumentos fueron enfermeras, aunque podría estar sesgada la información debido a que los grupos de enfermería son numerosos; habría que hacerse un estudio más específico o darle continuidad a éste para detectar más a fondo el porqué de estos resultados.

La violencia de género para Aguilar ${ }^{12}$ es una problemática social que afecta a mujeres de diferentes edades, clases sociales, culturas o niveles académicos y que supera estereotipos existentes respecto a quienes la sufren, por qué y dónde se produce. En este contexto es compatible con nuestros resultados.

Coincidimos con Givaudan ${ }^{13}$ en que debe tomarse en cuenta que en muchas escuelas no existe aún la disposición o los recursos para ofrecer talleres de prevención de larga duración enfocados a esta problemática. Por ello, resulta imperioso contar con estrategias breves que lleven a largo plazo a cambios de conductas en los jóvenes. El primer paso para lograr la prevención es la sensibilización en torno a esta problemática, lo cual permite empezar un proceso de cambio de normas sociales sobre el tema. Datos en las cuales se tendrán como sugerencias para la problemática de la presente investigación.

En otro estudio ${ }^{14}$ encontraron que totalidad de las mujeres estudiadas fueron víctimas de violencia de género, predominando la violencia de tipo psicológica $(100 \%)$, seguida de la violencia física $(98.6 \%)$ y finalmente la violencia sexual $(79.7 \%)$, lo cual coincide con nuestros resultados en los elevados índices de violencia que sufren las mujeres en la actualidad. Viviescas Vargas y cols $^{15}$ reportaron que el $28,81 \%$ dijo ser víctima de violencia de la pareja actual. La violencia emocional presenta la mayor prevalencia y la violencia física la mayor severidad. Estamos de acuerdo con que la prevalencia de la violencia psicológica es la más alta, pero no en que la severidad es mayor en la violencia física, también la reportamos en la psicológica.

Los hallazgos de nuestro estudio deberían interpretarse con prudencia, debido al tamaño reducido de la muestra estudiada. Sin embargo, es necesario continuar con esta línea de investigación para establecer estrategias de cobertura amplia que ayuden a disminuir esta problemática. 
La mayoría de jóvenes universitarias, futuras profesionales de la salud, han experimentado algún tipo de violencia a lo largo de su relación de pareja en los últimos 12 meses, ya sea violencia psicológica, que es la de mayor prevalencia con un índice de severidad que va de leve a grave; física, que es la segunda con mayor prevalencia aunque con índice de severidad leve en su mayoría; económica y/o sexual, que sólo un porcentaje pequeño la sufren, pero no menos importante por las repercusiones de llegar a estos tipos de violencia menos comunes y más reprochables en la sociedad, por lo menos en un índice de severidad leve.

Es necesario profundizar en los diferentes aspectos culturales, socioeconómicos, familiares y étnicos que influyen en la percepción de daño o de la tolerancia de ciertos actos ejercidos en su contra. Los resultados obtenidos que nos muestran maltrato o violencia hacia la mujer también sugieren la necesidad de desarrollar intervenciones efectivas para la prevención de la violencia de pareja que ayuden a sensibilizar a las jóvenes, así como proporcionar servicios de orientación e intervención a aquellas que ya hayan sufrido maltrato.

\section{Bibliografía}

1. INEGI. [Online]. 2011 [cited 2014 JUNIO 22. Available from: http://www.inegi.org.mx/prod_serv/contenidos/espanol/bvinegi/productos/estudios/sociodemografico/mujeresrural/2011/702825048327. pdf.

2. Rodríguez-Borrego María-Aurora, Vaquero-Abellán Manuel, Rosa Liana Bertagnolli da. Estudio transversal sobre factores de riesgo de sufrir violencia por compañero íntimo en la mujer enfermera. Rev. Latino-Am. Enfermagem [Internet]. 2012 Feb; 20( 1 ): 11-18. Available from: http://www.scielo.br/scielo.php?script =sci_arttext\&pid=S0104$11692012000100003 \& \operatorname{lng}=\bar{e}$. $\quad$ http://dx.doi. org/10.1590/S0104-11692012000100003.

3. Rodríguez Borrego $M A$, Vaquero Abellán $M$, Bertagnolli L, Muñoz-Gomariz E, Redondo-Pedraza R,

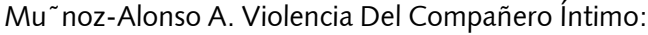
Estudio Con Profesionales De Enfermería Available From: Http://Www.Sciencedirect.Com/Science/Article/Pii/S0212656710004464.

4. Canaval GE, Humphreys J, De León N, González S, González MC. Pesquisa em base de datos. [Online].; 2009 [cited 2014 junio 24. Available from: http://bases.bireme.br/cgi-bin/wxislind.exe/iah/ online/? IsisScript $=$ iah/iah. $x i s \& s r c=$ google $\&$ base $=\mathrm{LI}$ LACS\&lang $=p \&$ nextAction $=$ Ink\&exprSearch $=5349$ 74\&indexSearch $=$ ID.

5. Valdez-Santiago Rosario, Híjar-Medina Martha C, Salgado de Snyder V Nelly, Rivera-Rivera Leonor, Ávila-Burgos Leticia, Rojas Rosalba. Escala de violencia e índice de severidad: una propuesta metodológi- ca para medir la violencia de pareja en mujeres mexicanas. Salud pública Méx [revista en la Internet]. 2006 Ene; 48( Suppl 2): s221-s231. Disponible en: http://www.scielo.org.mx/scielo.php?script $=$ sci_ arttext\&pid $=$ S0036-36342006000800002\&lng $=\mathrm{es}^{-}$

6. González S, González M C, De León N, Canaval G E, Humphreys J, Violencia de pareja y salud de las mujeres que consultan a las comisarías de familia, Cali, Colombia. Investigación y Educación en Enfermería 2009;27:209-217. Disponible en: http://www.redalyc.org/articulo.oa?id=105213195006.

7. Instituto Nacional de Salud Pública. (2006). Encuesta Nacional sobre Violencia contra las Mujeres. Recuperado el 01 de febrero de 2016, de http:// cedoc.inmujeres.gob.mx/documentos_download/ ENVIM_2006.pdf.

8. Calvo González Germán, Camacho Bejarano Rafaela. La violencia de género: evolución, impacto y claves para su abordaje. Enferm. glob. [Internet]. 2014 Ene ; $13(33$ ): 424-439. Disponible en: http://scielo. isciii.es/scielo.php?script $=$ sci_arttext\&pid $=$ S169561412014000100022\&lng $=\mathrm{es}^{-}$.

9. WHO multi-country study on Women's Health and Domestic Violence. 2005 Disponible en http://www. who.int/gender/violence/who_multicountry_study/ summary_report/summary_report_English2.pdf.

10. Vázquez $\bar{F}$, Otero $P$, Torres $A$, Blanco $V$, López $M$, Prevalencia y factores de riesgo de la violencia contra la mujer en estudiantes universitarias españolas. Psicothema 201022196-201. Disponible en: http:// www.redalyc.org/articulo.oa?id $=72712496004$.

11. García-Díaz V, Fernández-Feito A, RodríguezDíaz FJ, López-González ML, Mosteiro- Díaz MP, 
Lana-Pérez A.Violencia de género en estudiantes de enfermería durante sus relaciones de noviazgo, Revista Atención Primaria. aprim.2012.11.013.

12. Aguilar Ródenas $C$, Melgar Alcatud P, Alonso Olea $M$ J, Molina Roldán S, Violencia de género en el ámbito universitario. Medidas para su superación. Pedagogía Social. Revista Interuniversitaria 2009 85-94. Disponible en: http://www.redalyc.org/articulo. oa?id=135012677006. Fecha de consulta.

13. Givaudan $M$, Leenen I, Prado A, Pick S, "Yo quiero, yo puedo...prevenir la violencia»: Programa breve de sensibilización sobre violencia en el noviazgo. Salud Mental 201033153-160. Disponible en: http://www. redalyc.org/articulo.oa?id $=58215623006$. Fecha de consulta: 6 de octubre de 2016.

14. Navarro P. Violencia de Género, Autoestima y Consumo de Alcohol en Mujeres. Thesis 2011? https:// www.researchgate.net/publication/272157713_Violencia de Genero Autoestima y Consumo de Alcohol_en_Mujeres. DOI: 10.13140/2.1.4791.3760.

15. Viviescas-Vargas D P, Herrera-Trujillo $M$, Uicab-Pool G, Hidalgo-Solórzano E, Balam-Gómez M, López-López E, Idrovo A J, Cobertura efectiva del manejo de la violencia contra mujeres en municipios Mexicanos: límites de la métrica. Revista da Escola de Enfermagem da USP 201347781-787. Disponible en:

http: / / www.redalyc.org/articulo. oa? id $=361033327002$. 